\title{
A Rare Intronic Variation of Presenilin-1 (rs201992645) is Associated with Alzheimer's Disease and Down Syndrome Birth
}

\author{
Pranami Bhaumik ${ }^{1}$, Priyanka Ghosh ${ }^{1}$, Sujoy Ghosh ${ }^{2}$, Kausik Majumdar $^{3}$, Sandip Pal ${ }^{4}$, Biswanath Sarkar ${ }^{5}$, AbhishiktaGhosh Roy ${ }^{5}$, Abhinandan Chakraborty $^{1}$ \\ and Subrata Kumar Dey ${ }^{1 *}$ \\ ${ }^{1}$ Human genetics Research Unit, Department of Biotechnology, School of Biotechnology and Biological Sciences, West Bengal University of Technology, India \\ ${ }^{2}$ Genetics \& Genomics Research Unit, Department of Zoology, University of Calcutta, (Ballygunge Science college campus), India \\ ${ }^{3}$ Department of Elderly Medicine, National Neurosciences Centre/Peerless Hospital, Panchasayar, India \\ ${ }^{4}$ Department of Neurology, Burdwan Medical College, India
}

${ }^{5}$ DNA laboratory, Anthropological Survey of India, 27 Jawaharlal Nehru Road Kolkata, India

*Corresponding author: Subrata Kumar Dey, Human genetics Research Unit, Department of Biotechnology, School of Biotechnology and Biological Sciences, West Bengal University of Technology, BF - 142, Salt Lake City, Sector I. Kolkata, West Bengal, India, Tel: +919830278216; Fax: (033) 2334 1030; subrata.humangenetics@gmail.com

Rec Date: Jun 15, 2014, Acc Date: Sep 30, 2014, Pub Date: Oct 08, 2014

Copyright: ( 2014 , Bahumik P et al. This is an open-access article distributed under the terms of the Creative Commons Attribution License, which permits unrestricted use, distribution, and reproduction in any medium, provided the original author and source are credited.

\section{Abstract}

Background and objective: Presenilin-1 (PSEN-1) gene is a potent candidate that relates Alzheimer's disease (AD) to Down syndrome (DS). Genetic variation of PSEN-1 could be a risk factor that predisposes individual for both $A D$ and $D S$ and may contribute the common etiology of both the disorders.

Methods: We sequenced exon 8 of PSEN-1 with flanking introns in 136 DS patients with their parents, 96 AD patients, 173 age-matched controls. Results were analysed in-silico to anticipate the damaging effect at molecular level.

Results: A rare polymorphism rs201992645 was identified within intron 8 and in silico analysis revealed the variation as 'potentially damaging' at the transcript splicing level. The genotypic frequencies of mutant heterozygotes were $0.031,0.029$ and 0.029 for AD, DS and mother of DS respectively.

Conclusions: We have suggested that this variation may cause AD manifestation in mothers of DS patients and is the potential marker for predisposition testing of both disorders.

Keywords: Presenilin-1; Down syndrome; Alzheimer disease; Chromosomal segregation

\section{Introduction}

Alzheimer's disease (AD) and Down syndrome (DS) probably share a common genetic factor, as suggested by their frequent cooccurrence. $\mathrm{AD}$ like neuropathology such as $\beta$-amyloid plaque and neurofibrillary tangles are seen in virtually all DS patients at about 35 to 40 years of age [1]. Families with AD patient exhibit frequent birth of $\mathrm{DS}$ child; vis-a-vis, higher incidence of $\mathrm{AD}$ has been reported among the relatives of DS [2]. Usually the younger mothers bearing DS child are most likely to develop AD phenotype at their older age as evident in the study by Schupf et al. [3]. These observations led the researchers to hypothesize that frequent co-occurrence of $\mathrm{AD}$ and $\mathrm{DS}$ in families may originate from common genetic risk factor. In search of such risk agent researchers have identified Presenilin -1 gene (PSEN-1), located on chromosome 14, as a promising candidate which may relate AD and DS [4]. The PSEN-1 encodes a protein component of gamma-secretase complex which is involved in the processing of amyloid precursor protein (APP). $P S E N-1$ protein is involved in many cardinal mechanisms of several molecular pathways which when impaired lead to the manifestation of AD. The pathways include APP processing [5], notch signaling [6], neuronal apoptosis [7], mitochondrial dysfunction [8], calcium homeostasis and synaptic function [9]. This protein is also localized at centromeres, nuclear envelope of dividing cells, in kinetochores at interphase and is involved in the process of faithful chromosome segregation [10]. Mutation in PSEN-1 leads to chromosomal instability and trisomy 21 mosaicism among AD patients [11]. The study of Petersen et al. [12], revealed an association between the intronic polymorphism of PSEN-1 and meiotic nondisjunction of chromosome 21(Ch21) among the women bearing DS children. In present study we explored the sequence of PSEN-1 exon 8 to find out any mutation or polymorphism that might explain the role of this gene in common etiology and cooccurrence of $\mathrm{AD}$ and $\mathrm{DS}$ in the Indian Bengali population. We chose the exon 8 and its flanking intronic sequence, as this region is known to carry mutational hotspots and many mutations within this region exhibit association with $\mathrm{AD}[11]$.

\section{Materials and Methods}

\section{Study subjects}

Blood samples were collected from 96 probable Alzheimer's patients (mean age $62.26 \pm 11.93$ years with $70.48 \%$ male and $29.52 \%$ female subjects), 173 age matched healthy controls and 136 DS family trios (DS and parents) after obtaining their prior consent. Ethnic and 
Citation: Bhaumik P, Ghosh P, Ghosh S, Majumdar K, Sarkar B, et al. (2014) A Rare Intronic Variation of Presenilin-1 (rs201992645) is Associated with Alzheimer's Disease and Down Syndrome Birth. Hereditary Genet 3: 136. doi:10.4172/2161-1041.1000136

Page 2 of 4

demographic similarities were maintained between cases and controls through careful recruitment from hospitals within the same geographical localities. All participants were Bengali-speaking.

Dementia status of patients were diagnosed using DSM -IV (Diagnostic and Statistical Manual of Mental Disorders, Fourth Edition) criteria and NINCDS-ADRDA (National Institute of Neurological and Communicative Disorders and Stroke and the Alzheimer's Disease and Related Disorders Association) criteria, both of which are generally used for diagnosis of Alzheimer's disease by physicians. Severity of Dementia was measured by the MMSE (Mini Mental State Examination) score. Recruited controls were free from any recognizable neurodegenerative disorders.

\section{Mutation analysis}

Genomic DNA was isolated from blood using a Qiagen QIAamp DNA Blood Midi Kit (Catalogue No. 51185). A Polymerase Chain Reaction (PCR) was performed using oligonucleotide primer that has been described elsewhere [13]. The PCR amplicons were sequenced through ABI PRISM 3700 DNA Analyzer platform. The obtained sequence was thoroughly compared against the published genomic sequence (NCBI accession number NG_007386.2) of PSEN-1. The genotyping of APOE was done in all subjects and controls by direct DNA sequencing. The identified variation was submitted to NCBI (snp-sub@ncbi.nlm.nih.gov) to get its validity and a submitter SNP (ss) number (NCBI ss515119316) and reference SNP (rs) ID (rs201992645) were achieved in the process.

\section{Functional prediction of detected variation}

The online software 'Human Splicing Finder' Version 2.4.1 (http:// www.umd.be/HSF/), 'SpliceAid' (http://www.introni.it/splicing.html) and 'mutation t@sting' (http://www.mutationtaster.org/) were used to predict the probable damaging effects of the mutant allele at splicing level and protein expression levels.

\section{Results and Discussion}

We found a rare polymorphism rs201992645 [(NG_007386.2:g. 66696T $>$ A or NM_000021.3:c.868+37T >A) ss515119316] within the intron 8 of PSEN-1 gene (Figure 1) at the 73664874th nucleotide position on chromosome 14 (GRCh37.p5 Assembly). The change involves nucleotide transition $\mathrm{T}>\mathrm{A}$. The mutant ' $\mathrm{A}$ ' allele exists only as heterozygous condition (T/A) with genotype and allele frequencies of 0.031 and 0.02 respectively for $\mathrm{AD}$ cases and 0.029 and 0.01 respectively for DS. Within the parents of DS, the mutant allele was recorded only among the mother with genotypic and allelic frequencies of 0.029 and 0.01 respectively. But the father of the DS and controls were wild type homozygote $(\mathrm{T} / \mathrm{T})$. Statistical significance of the result was obtained by Fisher's exact test (mutated AD + DS cases/ total $\mathrm{AD}+\mathrm{DS}$ cases vs. mutated control individual/total control individual $=7 / 232$ vs. $0 / 173=\mathrm{p}$ value 0.02 ). The genotypes were not in an $\mathrm{H}-\mathrm{W}$ equilibrium, as we did not find any AA mutant homozygous among the participating families.

The women who bear mutant allele exhibited lower age at conception of DS foetus from the control [(mean \pm SD) $22.5 \pm 1.29$ vs. $32.02 \pm 3.64 ; \mathrm{p}$ value 0.045 ], as well as from the mothers of DS (32.42 \pm 3.53 ; $\mathrm{p}$ value 0.037 ) who did not carry the mutant allele. This variation was not associated with APOE genotypic variations.
To anticipate the probable damaging effect of the detected intronic mutation on PSEN-1 expression and functions, we analyzed the mutation by in silico method, using three bioinformatics softwares.

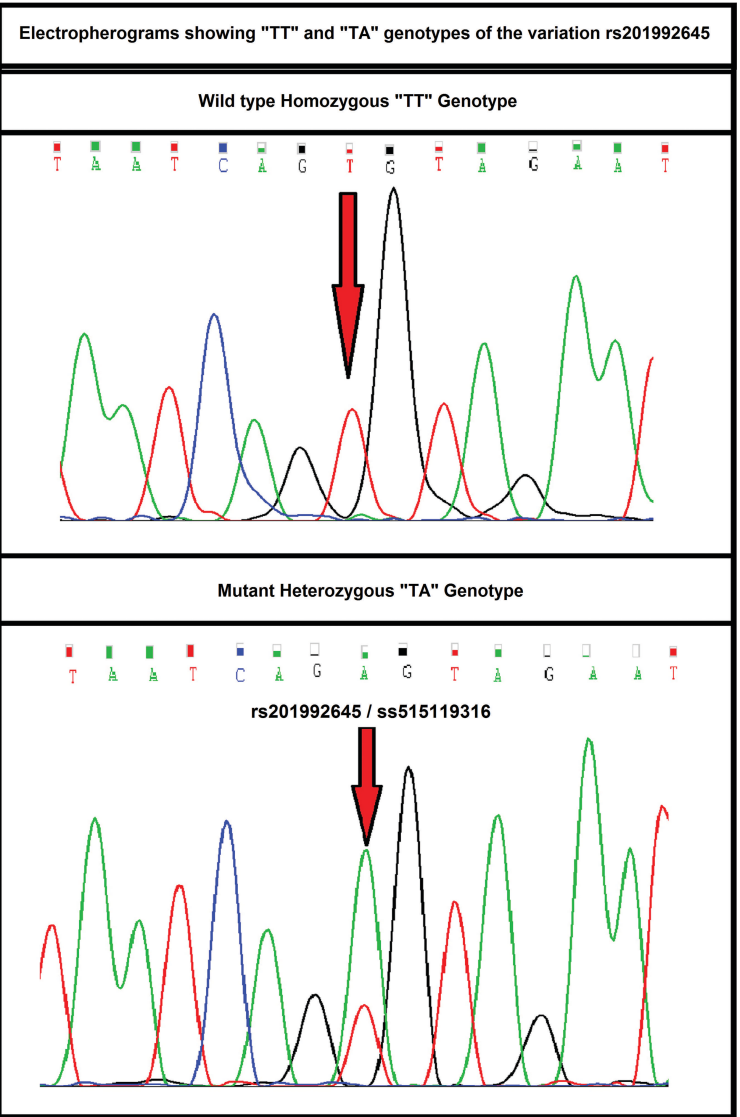

Figure 1: Electropherogram showing the found wild type homozygous "TT" genotype and mutant heterozygous "TA" genotype of the variation rs201992645 [(NG_007386.2:g.66696T >A or NM_000021.3:c.868+37T>A) ss515119316] on the intron-8 of PSEN-1 gene.

\section{Prediction of "human splicing finder" program}

The outcome of Human Splicing Finder program shows that the said neucleotide change (NG_007386.2:g.66696T $>$ A or NM_000021.3:c.868+37T $>A$ ) induces an alternation in acceptor splice site motif "tagtaatcagtgta" to a new splice site "tagtaatcagagTA" with $+69 \%$ variations. Potentially new branch point has been generated (CV for wild type and mutant are 40.01 and 69.64 respectively).The mutant allele also creates new binding site for essential sequence specific splicing factor protein SF2/ASF. Both RESCUE ESE hexamers matrix and intron-identity elements (EIEs) matrix show that a new enhancer motif is generated due to this SNP. Analysis of silencer motifs following the method by Sironi et al. [14] showed that motif- 1 is changed to a new site in mutant sequence. New site also formed for binding of splicing regulatory SR protein 9G8 as well as heterogeneous nuclear ribonucleoprotein hnRNP A1. (Supplementary Table SI). 
Citation: Bhaumik P, Ghosh P, Ghosh S, Majumdar K, Sarkar B, et al. (2014) A Rare Intronic Variation of Presenilin-1 (rs201992645) is Associated with Alzheimer's Disease and Down Syndrome Birth. Hereditary Genet 3: 136. doi:10.4172/2161-1041.1000136

Page 3 of 4

\section{Prediction of "SpliceAid" software program}

This database revealed that the said mutation rs201992645 generates ("gained') an additional SC35 protein binding site. Due to this transition mutation $(\mathrm{T}>\mathrm{A})$ in DNA sequence the corresponding RNA sequence "AGUAG" is changed to "UGUAG" which is the binding site for this protein (Figure 2). The SC35 is a $35 \mathrm{kDa}$ mammalian splicing factor which plays an important role in an alternative splicing of genes involved in both AD and DS [15]. This variation may help in splicing of intron-8 of PSEN-1gene.

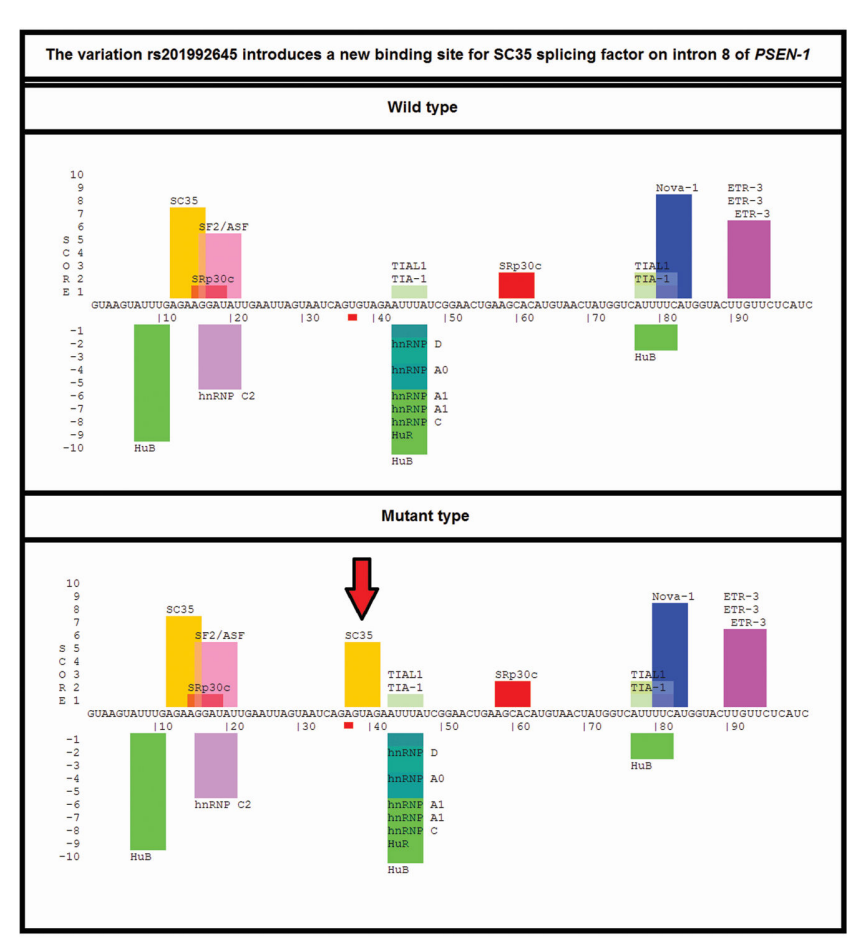

Figure 2: The outcome of the online software 'SpliceAid' (http:// www.introni.it/splicing.html): The Nucleotide variation rs201992645 [(NG_007386.2:g.66696T >A or NM_000021.3:c. $868+37 \mathrm{~T}>\mathrm{A})$ ss515119316] introduces a new binding site for a potent spicing factor "SC35". In wild type pre m-RNA sequence of PSEN-1 intron-8, the nucleotide stretch "UGUAG" is changed to "AGUAG" in mutant, which is the binding site for this SC35 protein involved in intron slicing.

\section{Prediction of “mutation t@sting” program}

The outcome of the program “mutation t@sting” suggested some alteration in the protein structure of PSEN-1 due to a splice site change. According to its prediction, this variation activates an additional splice site i.e. 'gained' at the genomic DNA position 61720 (score 0.71). Moreover, the existing splice site also gets stronger, i.e. "increased" at the position 61715 (score 0.78 for wild type vs. 0.97 for mutant output by the software). Potential cytoplasmic and transmembrane helical domains of PSEN-1 protein as well as the PAL motif required for normal active site conformation were predicted to get lost due to this variation. This variation may affect some important protein features as newly generated splice sites may lead to whole exon skipping. "mutation t@sting” also suggested that the variation may alter certain amino acid sequences, resulting in impaired gammasecretase activity, endoproteolytic cleavage, cleavage by caspase and progression of apoptosis. Both PKA and PKC mediated phosphorylation, interaction with CTNNB1, CTNND2 and MTCH1, production of amyloid-beta, processing of APP, NOTCH1 and CDH2 and disassembly of N-cadherin/PSEN-1 complex at the cell surface. Summary of the results is presented in Table 1.

\section{Conclusion}

The significance of our study lies in finding that the polymorphism rs201992645 (ss515119316) is strongly associated with both the AD and DS and the PSEN-1 gene is a prospective 'molecular link' between these two disorders. This polymorphism has not been described yet by any workers as simultaneous risk for NDJ of chromosome 21 and AD. In this regard our result is absolutely noble. We have anticipated through in silico approach, the prospective damaging effects of this nucleotide change in PSEN-1 on RNA transcript processing (Figure 2) as well as on protein conformation which might have some deleterious effect on various interacting subcellular pathways (Table 1; Supplementary Table 1). Considering the outcome of bioinformatics analyses, we can interpret that the said nucleotide change imparts pleiotropic effects on various interactive molecular pathways that may include both the signals for proper chromosome segregation and processing of amyloid precursor protein.

Presence of mutant allele only in the mothers of DS and DS child (not among the fathers of DS) is consistent with the notion that mothers of DS and DS patients usually susceptible to early onset of AD due to variation of PSEN-1. The mutation carrying mothers of DS had a much younger age of conception $[22.5 \pm 1.29$ years (mean \pm SD)] of the trisomy 21 fetus. This fact suggests that the nucleotide change in PSEN-1 predisposes the women simultaneously for Ch21 nondisjunction and $\mathrm{AD}$ irrespective of maternal age of conception. The said variation imparts maternal age independent risk of nondisjunction according to the 'DS risk model' proposed in previous studies [16,17]. Further longitudinal follow up study is needed to know whether mutant allele bearing DS develop $\mathrm{AD}$ phenotype later at their more advanced age. Studies are also needed to reveal whether this polymorphism is endemic for this Indian population or whether it affects ethnically different populations as well. Moreover, functional validation through experimentation is needed for anticipated in silico results. To conclude it can be said that the polymorphism rs201992645 (ss515119316) ( $\mathrm{T}>\mathrm{A})$ of PSEN-1 is a potential molecular marker for preconceptional genetic testing for screening DS pregnancy as well as to evaluate the risk of $\mathrm{AD}$ onset, particularly among the women.

\section{Acknowledgement}

We would like to thank all the AD patients, DS patients and their parents as well as the control individuals participating in the study.

\section{Financial Support}

This work was supported by the $<$ University Grants Commissions (UGC), New Delhi, India > under Grant <F. No. 36-299/2008 (SR)>. 
Citation: Bhaumik P, Ghosh P, Ghosh S, Majumdar K, Sarkar B, et al. (2014) A Rare Intronic Variation of Presenilin-1 (rs201992645) is Associated with Alzheimer's Disease and Down Syndrome Birth. Hereditary Genet 3: 136. doi:10.4172/2161-1041.1000136

Page 4 of 4

\begin{tabular}{|c|c|c|c|}
\hline $\begin{array}{l}\text { Inferred Change in Presenilin-1 } \\
\text { (PSEN-1) expression and its } \\
\text { product }\end{array}$ & $\begin{array}{l}\text { The interactive } \\
\text { genes }\end{array}$ & Sub-cellular processes/Molecular pathways & Probable Damaging effect \\
\hline \multirow{8}{*}{$\begin{array}{l}\text { The mutation might alter the } \\
\text { splicing pattern of the transcript; in } \\
\text { downstream it alters the protein } \\
\text { domain structure due to change in } \\
\text { amino acid sequence as the whole } \\
\text { exon may be skipped by splicing. }\end{array}$} & $\begin{array}{l}\text { APP } \\
\text { (Amyloid Precursor } \\
\text { Protein) }\end{array}$ & Involved in the amyloid plaque production in brain. & $\begin{array}{l}\text { Gamma secretase activity of PSEN-1 may be } \\
\text { changed, altering amyloid beta production. }\end{array}$ \\
\hline & Caspases & Involved in the proteolytic processing of PSEN-1. & $\begin{array}{l}\text { Metabolism of PSEN-1 via proteolytic processing } \\
\text { may be altered. }\end{array}$ \\
\hline & $\begin{array}{l}\text { PKC } \\
\text { (Phospho kinase C) }\end{array}$ & $\begin{array}{l}\text { Inhibits the caspase cleavage of PSEN-1 by } \\
\text { phosphorylating at amino acid residue } 346 \text {. }\end{array}$ & $\begin{array}{l}\text { Proteolytic processing of PSEN-1 and } \\
\text { progression of apoptosis may be altered. }\end{array}$ \\
\hline & $\begin{array}{l}\text { CTNNB1 } \\
\text { (Beta catenin) }\end{array}$ & Involved in regulation of apoptosis via cell signaling. & Neuronal apoptosis may be modulated. \\
\hline & $\begin{array}{l}\text { CTNND2 } \\
\text { (Delta catenin) }\end{array}$ & $\begin{array}{l}\text { Critical protein for maintenance of neuronal structure } \\
\text { and function. }\end{array}$ & Neuronal apoptosis may be modulated. \\
\hline & $\begin{array}{l}\mathrm{CDH} 2 \\
\text { (Cadherin) }\end{array}$ & $\begin{array}{l}\text { The cadherin-catenin-presenilin- } 1 \text { complex triggers } \\
\text { synaptic loss and make neurones vulnerable to } \\
\text { apoptosis. }\end{array}$ & Neuronal apoptosis may be modulated. \\
\hline & $\begin{array}{l}\text { MTCH1 } \\
\text { (Mitochondrial carrier } \\
\text { homolog 1) }\end{array}$ & $\begin{array}{l}\text { Its over-expression is responsible for mitochondrial } \\
\text { depolarization and apoptosis. }\end{array}$ & Apoptosis may be altered. \\
\hline & $\mathrm{NOTCH}-1$ & $\begin{array}{l}\text { PSEN-1-Notch1 interaction is necessary for } \\
\text { neurogenesis. }\end{array}$ & Neurogenesis may be impaired. \\
\hline
\end{tabular}

Table 1: Summary of damaging effect of mutation rs201992645 (NG_007386.2:g.66696T>A or NM_000021.3:c.868+37T>A) (ss515119316) of PSEN-1 gene on subcellular processes as inferred from In Silico analysis using the program mutation t@sting.

\section{References}

1. Mann DM, Esiri MM (1989) The pattern of acquisition of plaques and tangles in the brains of patients under 50 years of age with Down's syndrome. J Neurol Sci 89: 169-179.

2. Yatham LN, McHale PA, Kinsella A (1988) Down's syndrome and its association with Alzheimer's disease. Acta Psychiatr Scand 77: 38-41.

3. Schupf N, Kapell D, Nightingale B, Lee JH, Mohlenhoff J, et al. (2001) Specificity of the fivefold increase in AD in mothers of adults with Down syndrome. Neurology 57: 979-984.

4. Potter H (1991) Review and hypothesis: Alzheimer disease and Down syndrome-chromosome 21 nondisjunction may underlie both disorders. American Journal of Human Genetics 48: 1192-1200.

5. Duff K, Eckman C, Zehr C, Yu X, Prada CM, et al. (1996) Increased amyloid-beta42(43) in brains of mice expressing mutant presenilin 1. Nature 383: 710-713.

6. Woo HN, Park JS, Gwon AR, Arumugam TV, Jo DG (2009) Alzheimer's disease and Notch signaling. Biochem Biophys Res Commun 390: 1093-1097.

7. Alberici A, Moratto D, Benussi L, Gasparini L, Ghidoni R, et al. (1999) Presenilin 1 protein directly interacts with Bcl-2. J Biol Chem 274: 30764-30769.

8. Trushina E, Nemutlu E, Zhang S, Christensen T, Camp J, et al. (2012) Defects in mitochondrial dynamics and metabolomic signatures of evolving energetic stress in mouse models of familial Alzheimer's disease. PLoS One 7: e32737.

9. Ho A, Shen J (2011) Presenilins in synaptic function and disease. Trends Mol Med 17: 617-624.
10. Li J, Xu M, Zhou H, Ma J, Potter H (1997) Alzheimer presenilins in the nuclear membrane, interphase kinetochores, and centrosomes suggest a role in chromosome segregation. Cell 90: 917-927.

11. Geller LN, Potter H (1999) Chromosome missegregation and trisomy 21 mosaicism in Alzheimer's disease. Neurobiol Dis 6: 167-179.

12. Petersen MB, Karadima G, Samaritaki M, Avramopoulos D, Vassilopoulos D, et al. (2000) Association between presenilin-1 polymorphism and maternal meiosis II errors in Down syndrome. Am J Med Genet 93: 366-372.

13. Sherrington R, Rogaev EI, Liang Y, Rogaeva EA, Levesque G, et al. (1995) Cloning of a gene bearing missense mutations in early-onset familial Alzheimer's disease. Nature 375: 754-760.

14. Sironi M, Menozzi G, Riva L, Cagliani R, Comi GP, et al. (2004) Silencer elements as possible inhibitors of pseudoexon splicing. Nucleic Acids Res 32: 1783-1791.

15. Qian W, Liang H, Shi J, Jin N, Grundke-Iqbal I, et al. (2011) Regulation of the alternative splicing of tau exon 10 by SC35 and Dyrk1A. Nucleic Acids Res 39: 6161-6171.

16. Oliver TR, Feingold E, Yu K, Cheung V, Tinker S, et al. (2008) New insights into human nondisjunction of chromosome 21 in oocytes. PLoS Genet 4: e1000033.

17. Ghosh S, Feingold E, Dey SK (2009) Etiology of Down syndrome: Evidence for consistent association among altered meiotic recombination, nondisjunction, and maternal age across populations. American Journal of Medical Genetics A 149: 1415-1420. 